\title{
Endocannabinoids and endocannabinoid-like molecules are present in foods, blood and ileal fluids from ileostomy subjects: insight into possible metabolic implications
}

\author{
Silvia Tagliamonte ${ }^{1}$, Chris I R. Gill ${ }^{2}$, Laura Kirsty Pourshahidi ${ }^{2}$, Mary Slevin ${ }^{2}$, Roger Lawther ${ }^{3}$, \\ Gloria O'Connor ${ }^{3}$, Rosalia Ferracane ${ }^{1}$ and Paola Vitaglione ${ }^{1}$ \\ ${ }^{1}$ Department of Agricultural sciences, University of Naples "Federico II", Portici, Italy, \\ ${ }^{2}$ Nutrition Innovation Centre for Food and Health, Centre for Molecular Biosciences, Ulster University, Coleraine, \\ United Kingdom and \\ ${ }^{3}$ Altnagelvin Area Hospital, Western Health and Social Care Trust, Londonderry, United Kingdom
}

\begin{abstract}
The endocannabinoid system is a lipid signalling system with several regulatory functions throughout the body including regulation of appetite, food intake, macronutrient metabolism, pain sensation, blood pressure, mood, cognition and immunity. It consists of endocannabinoids (ECs), their receptors and enzymes involved in their synthesis and degradation. The two best-characterized endocannabinoids are $\mathrm{N}$-arachidonoylethanolamide (AEA) and 2-arachidonoylglycerol (2-AG). They are ligands of cannabinoid receptors CB1 and CB2 which are located in the central nervous system (CNS) but also in in the enteric nervous system, in the liver and in the adipose tissue.

Several structural congeners of ECs including N-acylethanolamines (NAEs) such as oleoylethanolamine (OEA), linoleyethanolamine (LEA), and palmitoyletahanolamine (PEA), show similar mechanisms of action, tissue distribution as well as pathways of formation and breakdown. They are considered "endocannabinoid-like" molecules acting through receptors that are located both in CNS and in the gastro-intestinal tract mucosa such as the G-protein coupled receptor 119 (GPR119) and peroxisome proliferatoractivated receptors (PPARs). NAEs display $\mathrm{EC}_{50}$ values for human GPR119 and PPAR- $\alpha$ between $65 \mathrm{ng} / \mathrm{mL}$ and $1000 \mathrm{ng} / \mathrm{mL}$. Some evidence indicated that NAEs, their phosphorylated precursors N-acylphosphatidylethanolamines (NAPEs) and ECs are also present in food. Thus, we developed a food database of these molecules and we calculated the daily dietary intake in a healthy population.

This study aimed to evaluate whether the concentrations of NAPEs, NAEs and ECs in the human intestinal lumen may support their activity through the receptors lining in the gastro-intestinal tract and if they correlated with those in plasma.

The observational study (16/NI/0267, Ulster University) involved 35 ileostomists (18F/17M, aged $18-70$ y, BMI $\left.17-40 \mathrm{~kg} / \mathrm{m}^{2}\right)$ who collected overnight fasting samples of ileal fluid and plasma. The concentrations of NAEs, NAPEs and ECs in biological samples were determined by LC-HRMS.

Data showed that NAEs and NAPEs were present in ileal fluids and plasma from all subjects ranging between 46851.0-104742.8 $\mathrm{ng} / \mathrm{mL}$ and $0.3-59.6 \mathrm{ng} / \mathrm{mL}$ in ileal samples and $1159.4-3985.7 \mathrm{ng} / \mathrm{mL}$ and $0.19-1.24 \mathrm{ng} / \mathrm{mL}$ in plasma, respectively. Contrarily, no ECs in ileal fluids were found except $2-\mathrm{AG}$ in two ileal samples whereas they ranged between $1.6-22.3 \mathrm{ng} / \mathrm{mL}$ in $\mathrm{plasma}$. Differences between genders and associations of plasma levels with individual energy intakes were found.

Altogether, the data demonstrated that NAEs in the intestinal lumen are sufficient to elicit metabolic responses through the gastrointestinal receptors.
\end{abstract}

\section{Conflict of Interest}

There is no conflict of interest 